\title{
An innovative way to highlight the power of each polymorphism on elite athletes phenotype expression
}

\author{
Valentina Contrò (1), Gabriella Schiera (2), Antonino Abbruzzo (1), Antonino Bianco \\ (3), Alessandra Amato (3), Alessia Sacco (3), Alessandra Macchiarella (3), Antonio \\ Palma (3), Patrizia Proia (3) \\ (1) Department of Statistics, SEAS, University of Palermo; (2) Department of Biological \\ Chemical and Pharmaceutical Sciences and Technologies (STEBICEF), University of Palermo; \\ (3) Department of Psychological, Pedagogical and Educational Sciences, Sport and Exercise \\ Sciences Research Unit, University of Palermo, Italy.
}

This article is distributed under the terms of the Creative Commons Attribution Noncommercial License (CC BY-NC 4.0) which permits any noncommercial use, distribution, and reproduction in any medium, provided the original author(s) and source are credited.

\begin{abstract}
The purpose of this study was to determine the probability of soccer players having the best genetic background that could increase performance, evaluating the polymorphism that are considered Performance Enhancing Polymorphism (PEPs) distributed on five genes: PPAR $\alpha$, PPARGC1A, NRF2, ACE e CKMM. Particularly, we investigated how each polymorphism works directly or through another polymorphism to distinguish elite athletes from non-athletic population. Sixty professional soccer players (age $22.5 \pm 2.2$ ) and sixty healthy volunteers (age $21.2 \pm 2.3$ ) were enrolled. Samples of venous blood was used to prepare genomic DNA. The polymorphic sites were scanned using PCR-RFLP protocols with different enzyme. We used a multivariate logistic regression analysis to demonstrate an association between the five PEPs and elite phenotype. We found statistical significance in NRF2 (AG/GG genotype) polymorphism/soccer players association $(\mathrm{p}<0.05)$ as well as a stronger association in ACE polymorphism $(\mathrm{p}=0.02)$. Particularly, we noticed that the ACE ID genotype and even more the II genotype are associated with soccer player phenotype. Although the other PEPs had no statistical significance, we proved that some of these may work indirectly, amplifying the effect of another polymorphism; for example, seems that PPAR $\alpha$ could acts on NRF2 (GG) enhancing the effect of the latter, notwithstanding it had not shown a statistical significance.

In conclusion, to establish if a polymorphism can influence the performance, it is necessary to understand how they act and interact, directly and indirectly, on each other.
\end{abstract}

Key Words: Polymerase chain reaction-restriction fragment length polymorphism, performance-enhancing polymorphisms, performance

Eur J Transl Myol 28 (1): 87-92, 2018

The abilities of athletes to stand out in particular physical qualities are determined, among other things, by adaptation to stress of circulatory, respiratory, muscular and other biological systems. The effectiveness of these mechanisms can certainly be improved with training. Biologically, it joins changes in tissues and cells, in turn depending on local variations of gene expression. The presence of specific gene variants decides the prowess of some physical traits, such as speed, muscle strength, greater possibility of injury and even emotional control. ${ }^{1,2}$ Invaluable contributions were provided by both the Human Genome Project, ${ }^{3}$ that sequenced 20.000 to 25.000 genes, and subsequently by the International HapMap Project, that identified variations in the human genome and genes related to common diseases such as asthma, cancer, diabetes and cardiovascular diseases. ${ }^{4}$

Brey et al. ${ }^{5}$ have instead developed a genetic polymorphisms map that may be associated with predispositions of better fitness and sports results. 239 fitness genes were identified: 7 in chromosomes X, 18 in mitochondrial DNA and 214 in autosomes.

Since several years, researchers are focusing their interest on research of the "right" genetic profile that can contribute to athletic performance and to determine the mechanisms that can guide a person to a specific athletic field. ${ }^{6-8}$ Contributions of genotype and phenotype to achieve elite performance is still not known. Adaptations to a resistance effort are strongly supported by the 
Table 1 Information on genotyping methods for each polymorphism

\begin{tabular}{|c|c|c|c|}
\hline GENE & PRIMERS & $\begin{array}{l}\text { TEMPERATURE } \\
\text { ANNEALING }\end{array}$ & $\begin{array}{c}\text { RESTRICTION } \\
\text { ENZYME }\end{array}$ \\
\hline \multirow{2}{*}{$\begin{array}{c}\text { PPARa intron } \\
\text { 7G/C }\end{array}$} & F 5'-3':TCACTCCTTAAATATGGTGG & \multirow[b]{2}{*}{$59^{\circ} \mathrm{C}$} & \multirow[b]{2}{*}{ TAQ I } \\
\hline & R 5'-3': AAGTAGGGACAGACAGGACCAGTA & & \\
\hline \multirow{2}{*}{$\begin{array}{l}\text { PPARgC1- } \\
\text { Gly482Ser }\end{array}$} & F 5'-3': TAAAGATGTCTCCTCTGATT & \multirow[b]{2}{*}{$50^{\circ} \mathrm{C}$} & \multirow[b]{2}{*}{ HPA II } \\
\hline & R 5'-3': GGAGACACATTGAACAATGAATAGGATTG & & \\
\hline \multirow{2}{*}{ ACE } & F 5'-3': GCCCTGCAGGTGTCTGCAGCATGT & \multirow[b]{2}{*}{$66^{\circ} \mathrm{C}$} & \multirow[b]{2}{*}{---} \\
\hline & R 5'-3': GGATGGCTCTCCCCGCCTTGTCTC & & \\
\hline \multirow{2}{*}{ CKMM } & F 5'-3': GGGATGCTCAGACTCACAGA & \multirow[b]{2}{*}{$50^{\circ} \mathrm{C}$} & \multirow[b]{2}{*}{ NCO I } \\
\hline & R 5'-3': AACTTGAATTTAGCCCAACG & & \\
\hline \multirow{2}{*}{ NRF2 AG/GG } & F 5'-3': AGTTTAGTGTCTCCCAGTGT & \multirow[b]{2}{*}{$50^{\circ} \mathrm{C}$} & \multirow[b]{2}{*}{ RSA I } \\
\hline & R 5'-3': CTTAGTTTTCTTGTATCCGT & & \\
\hline
\end{tabular}

mitochondrial functions: it is determined by genes of nuclear and mitochondrial DNA that encode enzymes of energy metabolism and are associated with aerobic physical fitness and insulin sensitivity, a factor that could play a key role in the pathophysiology of type 2 diabetes. For example, the genes coding for the peroxisome proliferator-activated receptor (PPAR) - delta and gamma coactivator 1 alpha, which will be discussed in details below, are very important for proper functions of mitochondria. PPAR $\delta$, in particular, regulates the expression of genes involved in lipid and carbohydrate metabolism and one of its functional polymorphism has been associated with a predisposition for an endurace performance. ${ }^{9}$

Nuclear respiratory factors NRF1 and NRF2 coordinate the expression of nuclear and mitochondrial genes relevant to mitochondrial biogenesis and cellular respiration. The polymorphism carriers in the opening translation sequence ATG of the gene NRF2 prove to have a greater run economy than non-carriers, which could potentially explain inter-individual differences in endurance capacity. ${ }^{10}$ PPARGC $1 \alpha$ is an important cofactor that regulates gene expression related to oxidative phosphorylation and ATP production in target tissues through coactivation of nuclear receptors. Studies in mice show that it increases performance during a test carried out at $\mathrm{VO}_{2}$ peak, showing an increase of oxidative peak capacity or greater oxygen consumption throughout the body. ${ }^{11}$ Studies have shown that PPARGC1A (Gly482Ser) polymorphism is associated with state athletic strength / power, and in particular, the PPARGC1A Ser / Ser genotype seems more favorable to athletes more powerful than controls. In these athletes there was an increase in the contribution of the anaerobic system to the production of energy needed for exercise. Athletes undergoing a heavyweight training program that had this genotype showed an advantage in developing resistance. ${ }^{12}$ The mitochondrial synthesis is stimulated by the pathway PPARGC1A (peroxisome proliferator- activated receptor coactivator 1 Gamma Alpha)-NRF (Nuclear Respiratory Factor)-TFAM (Mitochondrial Transciption Factor A). In summary, the receptor Peroxisome Proliferator-Activated Receptor- $\delta$ (PPAR- $\delta$ ) leads the promotion of PPARGC1A, which is the first stimulator of mitochondrial biogenesis. Factors NRF1 and NRF2 are intermediate transcription factors that stimulate the synthesis of TFAM, and the latter is the final effector that activates replication of mitochondrial DNA molecules. ${ }^{13}$. Among the factors that may affect this pathway, some genetic variations should be included, which can have effects individually or in combination with physical activity. For example, the functional polymorphism C294T (rs2016520) of the gene encoding PPAR- $\delta$ and Gly482Ser polymorphism (rs8192678) of the gene encoding PPARGC1A could play a key role in the activity of protein and/or mRNA. The $\mathrm{C}$ allele of the C294T polymorphism of PPAR- $\delta$ (site in exon 4 ) is associated with a highest transcriptional activity of the PPAR- $\delta$ promoter, inducing a binding site for the transcription factor $\mathrm{Sp}-1 .^{14}$ The minor allele of PPARGC1A Ser482 is associated with a more modest capacity of endurance during physical activity. ${ }^{15}$ The $G$ allele of the variant $A / G$ intron 3 of the subunits $\beta 1$ of the NRF2 gene (rs7181866) is associated with endurance and maximum oxygen consumption in response to resistance training. ${ }^{16}$

Common genetic variation that separates endurance athletes by sprinter, is probably due to natural selection. For example, the actin-binding protein alpha-actinin-3 (ACTN3) is highly present in the mechanical contraction of the fast muscle fibers; in sarcomere it is the largest component of the $\mathrm{Z}$ line and plays a fundamental organizing and regulating function for the muscle contraction. ACTN3 is nearly always expressed among professional athletes of power, ${ }^{17}$ while the polymorphism R577X (results in premature stop codon), which is associated with an absolute deficiency of ACTN3, it is prevalent to a greater extent among professional 

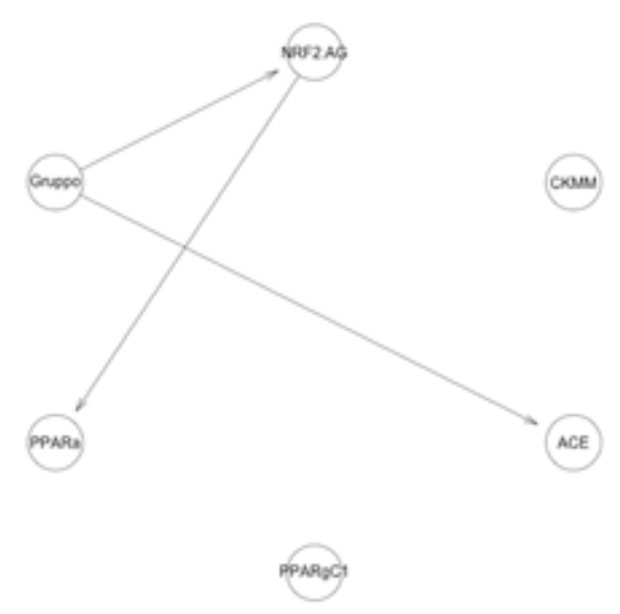

\section{Fig 1. Graphical model to evaluate the relative importance of each predictor to the regressors with regression type graphical models}

endurance athletes. ${ }^{18}$ In a recent work on soccer players it was showed a prevalence of ACTN3RR genotype, that was linked with a high production of ACTN3 and for the first time it was correleted with the training load and overall the mtDNA copy number increase was assess as a bioenergetics biomarker. ${ }^{19}$ Some authors observed a significantly lowest frequency of the $\mathrm{X} / \mathrm{X}$ genotype in power athletes compared with the control group. ${ }^{20}$ The gene Creatine kinase-MM (CK-MM) encoding the cytosolic muscle isoform of $\mathrm{CK}$, responsible for the rapid ATP regeneration during intense muscle contraction. ${ }^{21}$ Reduced expression of this enzyme may be responsible for muscle fatigue most likely due to increased intracellular concentration of inorganic phosphate. Moreover, studies of changes in CK-MM gene sequence showed a significant association between some of its polymorphisms and increased cardiorespiratory endurance, peak performance and lower decline in force generation; particularly, GG genotype and $\mathrm{G}$ allele are represented in power-oriented athletes. ${ }^{22}$ Several beneficial effects on athletic performance in endurance and sprint, were also observed in different genotypes of a single locus of angiotensin converting enzyme (ACE). The ACE gene, despite some controversy, seems to present variations that can be associated with many inherited traits, including physical, psychological and performance parameters, ${ }^{23}$ it has two alleles, called "I" and "D". The latter is associated with power-oriented athletes and anaerobic sports: the mechanism that underlies it is probably governed by the differences in strength gain at skeletal muscle level, because D allele was associated with greater increases in quadriceps strength after training, especially DD genotypes presented better performance for example during jump and sprint tests. In contrast, I allele could influence resistance performance improvements through a better use of substrate and muscle efficiency, with a consequent saving of energy supplies. On the other hand, DD genotypes may benefit athletes in activities that require strength and speed, while II ACE genotype in endurance activities. $^{24}$

\section{Materials and Methods}

Sixty sub-elite male Italian soccer players (age $22.5 \pm$ 2.2 ) and sixty sedentary male volunteers (age 21.2 \pm 2.3 ) were enrolled in this study. Athletes and controls were all Caucasian, in order to ensure the absence of a probable genetic ethnicity inclination and to overcome any problems of population stratification.

All participants gave their informed consent for genotyping, understood that the results would be anonymous and confidential. By standard clinical procedures venous blood samples were obtained, between 8:00 and 10:00 in the morning, at rest.

The samples were treated with anticoagulant (heparin) and used for genomic DNA extraction (PureLink Genomic DNA, ThermoFisher Scientific).

The polymorphic sites were analyzed by PCR-RFLP protocols with different enzymes (Tab 1).

\section{Results}

Using a multivariate logistic regression analysis, we tried to figure out if it is possible to speculate the impact of each polymorphism on the expression of elite soccer

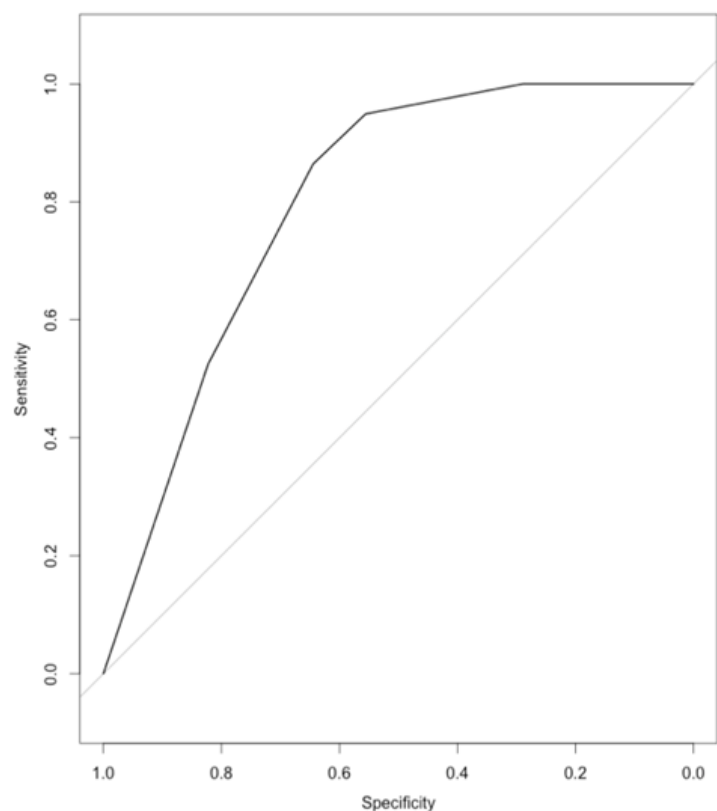

Fig 2. Receiver operating characteristic curve (ROC) summarizing the ability of elite genotype score to classify potential elite athletes from non-athletes (controls). AUC indicates the area under the curve $(95 \%$ confidence intervals). 
player phenotype. Particularly, we investigated how each polymorphism works directly or through other polymorphism to discern elite athletes from non-athletic population. We found statistical significance in NRF2 (AG/GG genotype) polymorphism/soccer player association $(p=0.03)$ as well as in ACE II genotype $(p=0.02)$. Particularly, it merges a probability three times higher to become soccer players if you have these two polymorphisms (NRF2 AG/GG and ACE II). Furthermore, despite PPAR $\alpha$ has not a statistical significance, have a positive estimate value $(0,5)$ compare to the others that suggest a possible involving in the induction of the expression of elite phenotype (Tab. 2). Although the others PEPs (PPAR $\alpha$, PPARGC1, CKMM) had no statistical significance and they didn't show any correlation with the others, it means they probably work independently. Figure 1 summarized all five polymorphisms belong to the performanceenhancing polymorphism family and how it's probably works. Previously, we demonstrated that PPAR $\alpha$ had a significant trend of association between GG polymorphism, especially $G$ allele, and elite soccer player. ${ }^{25}$ Through this new approach, we realized that the polymorphism investigation could be a marker of the elite phenotype only if it's associated each other. As a matter of fact PPAR $\alpha$ demonstrated a significant statistical association with soccer player group ( $p=0.03)$ only when is investigated with NRF2 genotype. Definitely PPAR $\alpha$ could acts on NRF2 (GG) enhancing the effects of the latter nonetheless it had not shown a statistical significance (Fig 1). The genotype distribution was in Hardy-Weinberg equilibrium for the whole group (soccer players and control group). The area under the ROC curve is .80 (AUC) that be considered to be "good" at separating elite soccer players from control group. The ROC analysis highlights a significant discriminating accuracy of the model in identifying an elite soccer players (area under the curve [AUC] $=0.80 ; 95 \%$ confidence interval [CI]: 0.71-0.88) (Fig 2). Definitely, the results of the statistical analysis for the comparison of elite athletes vs controls indicated that particularly 3 polymorphisms of those analysed were significantly associated with elite phenotype: 2 directly (NRF2 AG/GG and ACE II) and 1 indirectly (PPAR $\alpha$, through NRF2).

\section{Discussion}

Since many years disputes have emerged about the interpretation of association studies involving ACE gene (I/D). Some studies, indeed, related the I allele with power performance instead of endurance performance 26,27 .The novelty of this study lies in the fact that even in a team sport like football, it is possible to find this type of association. Particularly, we applied a multivariate linear regression to better analyzed which is the impact of each polimorphism alone or together to induce elite phenotype expression. Taken together the results obtained in our previous study ${ }^{25}$ and in this one, it is clear that the new approach shows power of each polymorphism as performance-enhancing factor. In the attempt to implement experimental designs reducing selection errors and the study of athletes from different sports, comparing them with a control group adequately

Table 2. Estimates and Standard Errors for the Model and the significance of each coefficient in the presence of the others

\begin{tabular}{|l|l|l|l|l|}
\hline VARIABLE & ESTIMATES & STD ERROR & Z VALUE & P-VALUE \\
\hline INTERCEPT & $-2,4614$ & 1,5752 & $-1,56$ & 0,1182 \\
\hline PPARa GC & $-0,1094$ & 0,7996 & $-0,14$ & 0,8912 \\
\hline PPARa GG & 0,5458 & 0,7942 & 0,69 & 0,4919 \\
\hline PPARgC1 SG & $-0,3521$ & 0,5198 & $-0,68$ & 0,4981 \\
\hline PPARgC1 SS & $-0,6604$ & 0,8459 & $-0,78$ & 0,4350 \\
\hline ACE ID & 0,6882 & 0,5347 & 1,29 & 0,1981 \\
\hline ACE II & 3,3034 & 1,5015 & 2,20 & $0,0278 *$ \\
\hline CKMM AG & $-0,8885$ & 0,5113 & $-1,74$ & $0,0822 \quad \cdot$ \\
\hline CKMM GG & $-0,8795$ & 0,7630 & $-1,15$ & 0,2490 \\
\hline NRF2 AG/AG & 3,1164 & 1,4368 & 2,17 & $0,0301 *$ \\
\hline NRF2 AG/GG & 3,8156 & 1,7672 & 2,16 & $0,0308 *$ \\
\hline
\end{tabular}


large, helps to make results achievable and reproducible. In details, during a game of 90 minutes, medium-high level players run for about $8-10 \mathrm{~km}$ at an intensity close to the anaerobic threshold (80-90\% of maximum heart rate). However, within this endurance context are required numerous flashes of explosive strenght, as kicks, jumps, sprints, changes of speed and direction and tackle, in addition to maintaining balance under defensive pressure to control the ball. Since the main component is, however, resistance, this could explain a genotypic profile typical of endurance athletes associated with football players. On the other hand, it should be stressed that athletic performances are multifactorial events: factors such as environment, gene-gene and geneenvironment interactions play valuable roles in the complex traits of champions, that can not be reduced to a set of genetic polymorphisms. ${ }^{28}$ This innovative method begins to be used in many other fields, such as the prevention of injuries and the investigation of muscular morphological changes, such as atrophy or myasthenic syndrome. $^{29,30,31}$

\section{List of acronyms}

ACE - angiotensin converting enzyme

ACTN3 -actin-binding protein alpha-actinin-3

CK-MM - Creatine kinase-MM

NRF - Nuclear Respiratory Factor

PCR-RFLP - Polymerase chain reaction-restriction fragment length polymorphism

PEPs - performance-enhancing polymorphisms

PPAR - peroxisome proliferator-activated receptor

TFAM - Mitochondrial Transciption Factor A

$\mathrm{VO}_{2}$ - oxygen uptake

\section{Author's contributions}

Each author contributed in equal part to the manuscript.

\section{Acknowledgments and Funding}

This research received no specific grant from any funding agency in the public, commercial or not-for-profit sectors.

\section{Conflict of Interest}

The authors declare no conflicts of interests derived from the outcomes of this stud.

\section{Ethical Publication Statement}

Institutional Review Board that approved the protocol for the study: Sport and Exercise Sciences Research Unit, University of Palermo, Italy.

We confirm that we have read the Journal's position on issues involved in ethical publication and affirm that this report is consistent with those guidelines.

\section{Corresponding Author}

Patrizia Proia, via Pascoli, 6, 90144 Palermo (Italy). Tel: +39 09123899919 E-mail: patrizia.proia@unipa.it

\section{E-mails of co-author}

Valentina Contrò: valecontro@gmail.com Gabriella Schiera: gabriella.schiera@unipa.it Antonino Abbruzzo: aabbruzzo@gmail.com Antonino Bianco: antonino.bianco@unipa.it Alessandra Amato: amale94@ hotmail.it Alessia Sacco: alessiasacco1989@gmail.com Alessandra Macchiarella: alemacchi89@gmail.com Antonio Palma: antonio.palma@unipa.it

\section{References}

Lippi G, Longo UG, Maffulli N. Genetics and sports. British Medical Bulletin 2009;93:27-47.

Jung H, Lee N, Park S. Interaction of ACTN3 gene polymorphism and muscle imbalance effects on kinematic efficiency in combat sports athletes. J Exerc Nutrition Biochem 2016;20:1-7.

Collins FS, Morgan M, Patrinos A. The human genome project: lessons from large-scale biology. Science 2003;11:286.

Thorisson GA, Smith AV, Krishnan L, Stein LD. The international HapMap project web site. Genome research 2005;15:1591-3.

Brey CW, Nelder MP, Hailemariam T, et al. Krüppel-like family of transcription factors: an emerging new frontier in fat biology. Int J Biol Sci 2009;5:622-36.

Grealy R, Herruer J, Smith CL, et al. Evaluation of a 7Gene Genetic Profile for Athletic Endurance Phenotype in Ironman Championship Triathletes. PLoS One 2015;30;10(12).

Santiago C, Ruiz JR, Muniesa CA, et al. Does the polygenic profile determine the potential for becoming a world-class athlete? Insights from the sport of rowing. Scand J Med Sci Sports 2010;20:e188-94.

Ruiz JR, Gómez-Gallego F, Santiago C, et al. Is there an optimum endurance polygenic profile? J Physiol 2009;587(Pt 7):1527-34.

Ahmetov II, Astranenkova IV, Rogozkin VA. Association of PPARD gene polymorphism with human physical performance. Mol Biol (Mosk) 2007;41,852-7. $\mathrm{He} \mathrm{Z}, \mathrm{Hu} \mathrm{Y}$, Feng L Lu Y, et al. NRF2 genotype improves endurance capacity in response to training. Int J Sports Med 2007;28:717-21.

Calvo JA, Daniels TG, Wang X, et al. Muscle-specific expression of PPAR gamma coactivator-1alpha improves exercise performance and increases peak oxygen uptake. J Appl Physiol 2008;104,1304-12.

Gineviciene V, Jakaitiene A, Aksenov MO, et al. Association analysis of ACE, ACTN3 and PPARGC1A gene polymorphisms in two cohorts of European strength and power athletes. Biol Sport 2016;33:199-206.

Kanki T, Ohgaki K, Gaspari M, et al. Architectural role of mitochondrial transcription factor $\mathrm{A}$ in maintenance of human mitochondrial DNA. Mol Cell Biol 2004;24:9823-34.

Skogsberg J, Kannisto K, Cassel TN, et al. Evidence that peroxisome proliferator-activated receptor delta influences cholesterol metabolism in men. Arterioscler Thromb Vasc Biol 2003;23:637-43. 
Lucia A, Gomez-Gallego F, Barroso I, et al. PPARGC1A genotype (Gly482Ser) predicts exceptional endurance capacity in European men. J Appl Physiol 2005;99:3448.

$\mathrm{He} \mathrm{Z,} \mathrm{Hu} \mathrm{Y,} \mathrm{Feng} \mathrm{L,} \mathrm{et} \mathrm{al.} \mathrm{NRF-1} \mathrm{genotypes} \mathrm{and}$ endurance exercise capacity in young Chinese men. Br J Sports Med. 2008;4:361-6.

Yang R, Shen X, Wang Y, et al. ACTN3 R577X Gene Variant is Associated with Muscle-related Phenotypes in elite Chinese Sprint/Power Athletes. J Strength Cond Res 2017;31:1107-15

Yang N, MacArthur DG, Gulbin JP, et al. ACTN3 genotype is associated with human elite athletic performance. Am J Hum Genet 2003;73:627-31.

Galeandro V, Notarnicola A, Bianco A et al. ACTN3/ACE genotypes and mitochondrial genome in professional soccer players'performance. J Biol Regul Homeost Agents 2017;31:207-13.

MacArthur DG, North KN. A gene for speed? The evolution and function of alpha-actinin-3. Bioessays 2004; 26:786-95.

Zhou DQ, Hu Y, Liu G, et al. Muscle-specific creatine kinase gene polymorphism and running economy responses to an 18-week 5000-m training programme. $\mathrm{Br}$ J Sports Med 2006;40:988-91.

He EP, Li YH, Qian JD, Yan HW. Association of CKMM gene $A / G$ polymorphism and athletic performance of Uyghur nationality. Chinese journal of applied physiology 2016;32:82-6.

Moran CN, Vassilopoulos C, Tsiokanos, et al. The associations of ACE Polymorphisms with physical, physiological and skill parameters in adolescents. Eur $\mathbf{J}$ Hum Genet 2006;14:332-9.

Dionísio TJ, Thiengo CR, Brozoski DT, et al. The influence of genetic polymorphisms on performance and cardiac and hemodynamic parameters among Brazilian soccer players. Appl Physiol Nutr Metab 2017;42:596604.

Proia P, Bianco A, Schiera G, et al. PPAR $\alpha$ gene variants as predicted performance-enhancing polymorphisms in professional Italian soccer players. Open Access J Sports Med 2014;5:273-8.

Tobina T, Michishita R, Yamasawa F, et al. Association between the angiotensin I-converting enzyme gene insertion/deletion polymorphism and endurance running speed in Japanese runners. Journal of Physiological Sciences 2010;60;5:325-30.

Offer A, Ruthie A, Chen Y, et al. The ACE deletion allele is associated with Israeli elite endurance athletes. Exp Physiol 2007; 92;5; 881-886.

Buxens A, Ruiz JR, Arteta D, et al. Can we predict toplevel sports performance in power vs endurance events? A genetic approach. Scandinavian Journal of Medicine \& Science in Sports 2011;21;4:570-9.

Bevilacqua JA, Lara M, Díaz J, Campero M, Vázquez J, Maselli RA. Congenital Myasthenic Syndrome due to DOK7 mutations in a family from Chile. Eur J Transl Myol. $2017 \quad$ Sep 20;27(3):6832. doi: 10.4081/ejtm.2017.6832. eCollection 2017 Jun 27.

Seene T, Umnova M, Kaasik P. Morphological peculiarities of neuromuscular junctions among different fiber types: Effect of exercise. Eur J Transl Myol. 2017 Jun 27;27(3):6708. doi: 10.4081/ejtm.2017.6708. eCollection 2017 Jun 27.

Hockerman GH, Dethrow NM, Hameed S, et al. The Ubr2 Gene is Expressed in Skeletal Muscle Atrophying as a Result of Hind Limb Suspension, but not Merg1a Expression Alone. Eur J Transl Myol. 2014;24(3):3319. doi: 10.4081/ejtm.2014.3319. eCollection 2014 Sep 23.

Received for publication: November 10, 2017

Revision received: November 10, 2017

Accepted for publication: November 10, 2017 